\title{
Theoretical Studies of Heavy Ion Collisions via Holography
}

\author{
Irina Aref'eva ${ }^{1, *}$ \\ ${ }^{1}$ Steklov Mathematical Institute, Russian Academy of Sciences, Gubkina str. 8, 119991, Moscow, \\ Russia
}

Abstract. Applications of holographic bottom-up QCD models to the studies of Heavy Ion Collisions (HIC) are discussed.

\section{Introduction}

The schematic picture of HIC is presented in Fig.1. The local thermalization, which is expected to take place about $1 \mathrm{fm} / \mathrm{c}$ after collision, is accompanied by a huge entropy production. Physics here is not fully understood, since usual QCD calculations are not applicable to non-equilibrium processes in strong coupling regime. It is believes that the local thermalization produces initial conditions for the hydrodynamic evolution. In the freeze-out and post-equilibrium stage $\left(\tau>\tau_{0}\right)$ the final particles decouple and become free at some proper time $\tau_{f}>\tau_{0}$.

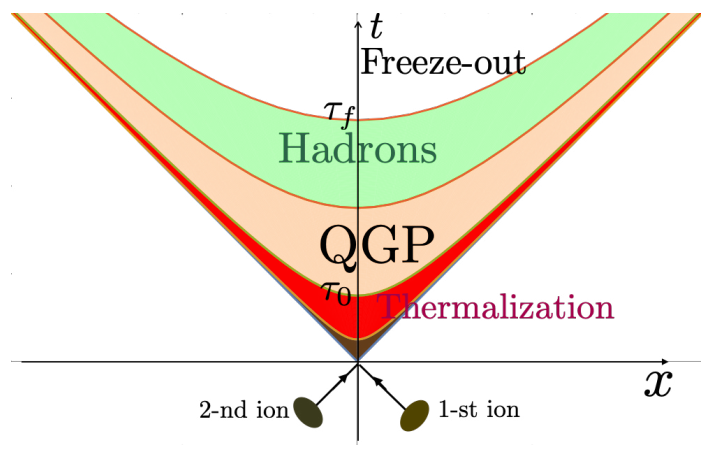

Figure 1. Characteristic time scales in HIC: in the initial or pre-equilibrium stage $\left(0<\tau<\tau_{0}\right)$ the two nuclei pass through each other; thermalization time is about $\tau_{0} \sim 1 \mathrm{fm} / \mathrm{c}=3.3 \cdot 10^{-24} \mathrm{~s}$; life-time of QGP is about $10 \mathrm{fm} / \mathrm{c}$ and after freeze-out time $\tau_{f}$ the final particles decouple.

Experimental studies of the collisions of heavy nuclei at relativistic energies have established the existence of the quark-gluon plasma (QGP) - a state of hot dense nuclear matter, in which quarks and gluons are not bound into hadrons. This state is characterized by the temperature $T$ and chemical potential $\mu$. With a decrease of chemical potential and temperature the matter goes to the confinement phase and QCD phase transition at $T=T(\mu)$ is expected.

\footnotetext{
*e-mail: arefeva@mi-ras.ru
} 
The experimental search for the QCD phase transition is nowadays one of the central goals for current and future collider facilities [1]. The experimental search is mainly related to the measurement of fluctuations of net-proton or net-charge multiplicity [2-5], which are expected to exhibit non-monotonic behavior near the phase transition. A proper understanding of the experimental results requires careful analysis of the dynamical processes that take place near phase transition lines. The expected phase diagram is presented in Fig.2.

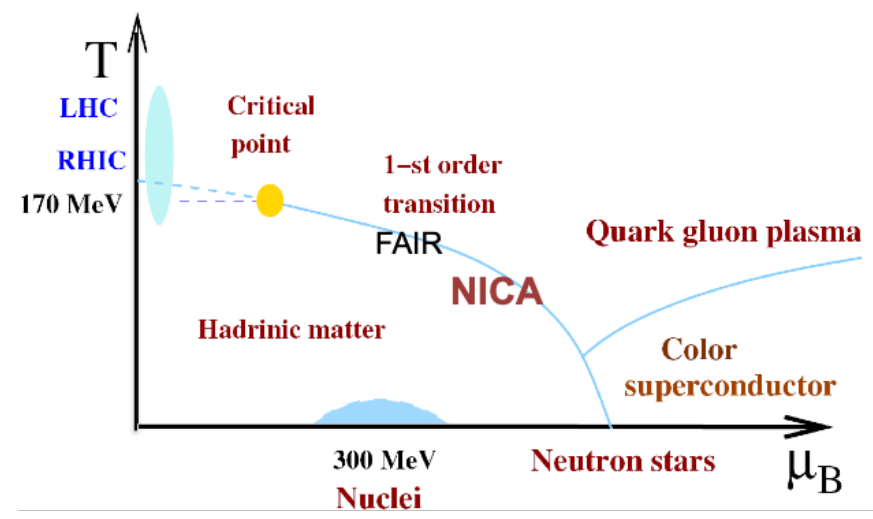

Figure 2. The expected QCD phase diagram.

There are several theoretical approaches to searches for QCD phase transitions. One of these approaches is the lattice approach [6,7], but there are difficulties with non-zero chemical potential calculations in there. Holographic duality (see few comments about holographic approach to QCD in Sect.2 and for review see [8-10]) provides an alternative approach to the study of QCD phase transitions.

Let us note that QGP behaves as a nearly inviscid fluid with initial spatial anisotropy [11]. So it is natural to study phase transition in the anisotropic QCD. There are also theoretical indications to deal with the anisotropic HQCD (see Sect.3). In the lattice approach, one can deal with anisotropic lattice. Some calculations have been performed in this direction [12]. The holographic approach also has a natural framework to deal with spatial anisotropy (see [13-15], comments in Sect.5 and results presented in talks at this conference [16, 17]).

\section{Holography as a new type of phenomenology}

Holography is nowadays one of the most effective tools to study quantum non-equilibrium physics of strongly interacting many body systems. These systems include ultrarelativistic heavy ion collisions, cold atom systems, quantum simulators, "ultrafast" techniques in condensed matter physics, etc. Holography translates the physics of quantum many body systems into a dual classical gravitational problem in a space-time with an extra dimension.

Holography is a new type of phenomenology. In the context of QCD this means that a phenomenological model, say HQCD (holographical QCD) has to describe QCD at all energy scales - from hight to low scales. The HQCD has to reproduce the usual QCD results at short distances (results obtained by perturbative theory) and Lattice QCD results at large distances (confinement etc.). At an intermediate energy scales and extremal conditions (hight density, or chemical potential) HQCD has to give new theoretical results, that are in agreement with the results of the experimental studies, or predict new results. HQCD has a 
support from exact dual holographic descriptions of more simple models, such as $\mathcal{N}=4$ supersymmetric Yang Mills theory at large N limit [18]. HQCD is formulated as 5-dimensional theory, where the 5-th coordinate takes a role of an energy scale. This 5-dim background is supposed to be a deformed version of 5-dimensional Schwarzschild-AdS, or ReissnerNordström Schwarzschild-AdS space time. It is supposed that in 5-dim background there is a dilaton field, whose dynamics in 5-dimension describes the running coupling in 4-dim quantum theory. So, schematically, we have the following picture.

- Relations between RG flows [19, 20].

- On the one hand we have is 5-dim background

$$
d s_{5}^{2}=w(z)\left(d s_{4}^{2}+d z^{2}\right)
$$

Here 5-th coordinate $z$ takes a role of an energy scale and we have the dilaton field $\phi=\phi(z)$ that defines the running coupling $\lambda$ in 4-dim theory

$$
\lambda(z)=\exp \phi(z)
$$

and holographic $\beta$-function as

$$
\beta(z)=\frac{d \lambda(z)}{d \log w(z)} .
$$

One introduces the field $X(z)$

$$
X(z)=\frac{\beta}{3 \lambda},
$$

that satisfies the first order non-linear differential equation

$$
\frac{d X}{d \phi}=\mathcal{X}(\phi, X)
$$

Equation (5) follows from the Einstein equation of motion for the background (1) [20] and it is related with the Hamiltonian form of the Einstein gravitational equations. The explicit form of function $\mathcal{X}(\phi, X)$ depends on the dilaton potential (see few examples in [21]).

- On the other hand we have 4-dim QCD. We know the QCD renormgroup flow of the running coupling

$$
\beta(E)=\frac{d \lambda(E)}{d \log E}
$$

perturbatively in UV and we also expect its form in IR from lattice calculations (see Fig.3). Note that the form of the renormgroup flow depends on the mass of quarks, number of flavours, temperature, chemical potential, etc.

Comparing (3) and (6) we see that $w$ plays a role of the energy scale. One of the first example of the dilaton potential that reproduces the QCD $\beta$-function up to first few order has been constructed in [10]. 


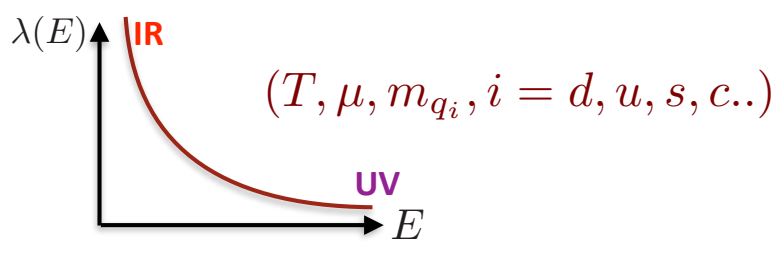

Figure 3. The schematic picture of QCD RG flow. The form of the curve dependes on quark's mass, number of flavours, temperature, chemical potential, etc.

- The form of the Cornell potential.

- From the 5-dim side: we can find temporal Wilson loops behavior in 5-dim backgrounds.

- From the 4-dim side: temporal Wilson loops behavior is the subject of intensive studies in lattice QCD at least at zero chemical potential for realistic case or at $N_{f}=2$ for non-zero chemical potentials.

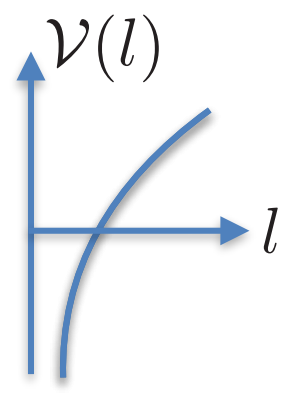

Figure 4. The schematic form of the Cornell potential, for details see [16].

- $(T, \mu)$ phase diagram.

- On the one hand we have holographic $(T, \mu)$ phase diagrams defined by the Wilson loops behavior in 5-dim backgrounds.

- On the other hand we know $(T, \mu)$ phase diagrams of Lattice QCD.

In Fig. 5 we see the different locations of the first order phase transitions.

There are two different strategies to find a suitable phenomenological model:

- start from a given 5-dim Lagrangian, in particular, from a given form of the dilaton action which reproduces QCD $\beta$-function, then find background by solving equation of motions and finally find the Cornell potential (in principal, one can add more matter fields to adjust these phenomenological data);

- start from a background that guaranties a suitable form of the QCD diagram and then find out the behaivior of the $\beta$-function. 


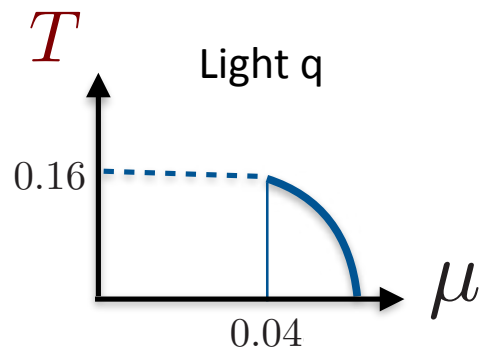

A

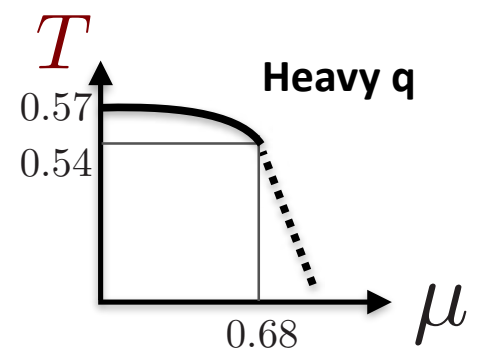

B

Figure 5. Holographic QCD phase diagrams: A) for light quarks and B) for heavy quarks. Sold lines correspond to the first order phase transitions and dashed lines correspond to the crossovers.

Both strategies are used in the literature. One of the goals of this talk is to advocate there is one more parameter that should be taken into account in the search of the phenomenological model. This parameter is nothing but the anisotropy parameter. To argue why there is a reason to deal with it we have to discuss the thermalization of a matter created in HIC. This is a subject of Sect.3.

\section{Thermalization and QGP formation in holographic approach}

According to the holographic approach the thermalization process in d-dimensional spacetime is understood as a black hole formation in the $(\mathrm{d}+1)$-dimensional background that is AdS or deformed AdS space-time. To initiate the process of $\mathrm{BH}$ formation one has to perturb the initial background metric. Within holographic approach this perturbation should be related with a perturbation of the energy-momentum tensor of the boundary theory. In the context of holographic application to HIC, one can make a perturbation of the $(d+1)$ dimensional background metric that near the boundary mimics the energy-momentum tensor corresponding to the heavy ion collision, a schematic picture presented at Fig.6.A.

It is interesting to check this proposal in exact integrable cases. The $A d S_{3}$ case, where the black hole formation may occur under collision of two ultra-relativistic particles, provides such a possibility. Schematically we have the picture presented at Fig.6.B. Thermalization in $(1+1)$-dimensional CFT after simultaneous generation of two high-energy excitations in the antipodal points on the circle has been studied in [22]. The holographic picture of such quantum quench is the creation of BTZ black hole from a collision of two massless particles. In [22], we have performed holographic computation of entanglement entropy in the boundary theory and analyse their evolution with time. We have shown that equilibration of the entanglement in the regions which contained one of the initial excitations is generally similar to that in holographic global quench models (Fig.8), but with some important distinctions (Fig. $7^{1}$, also see [22] for details).

\footnotetext{
${ }^{1}$ The advantage of studying thermalization after global quenching is that it is easy to generalize to higher spacetime dimensions, while generalizing bilocal quenching to cases of high dimensionality is more complicated (meaning the description of the formation of a black hole in the collision of shock waves).
} 


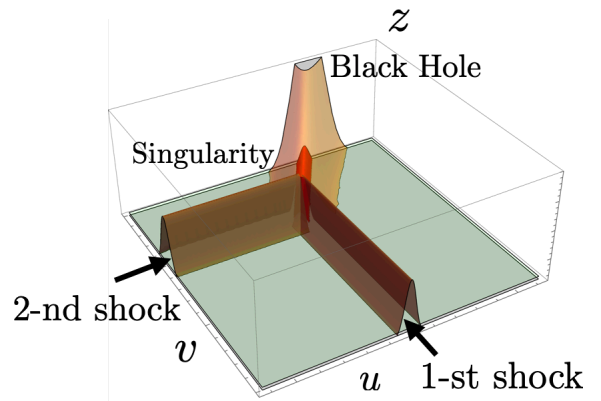

A

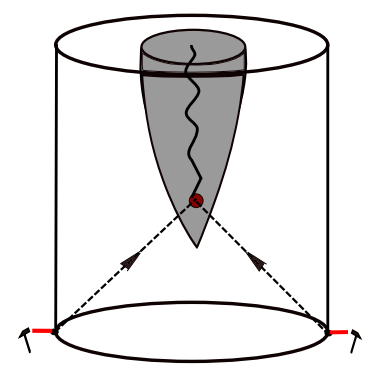

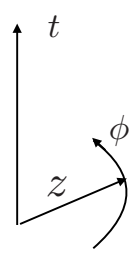

C

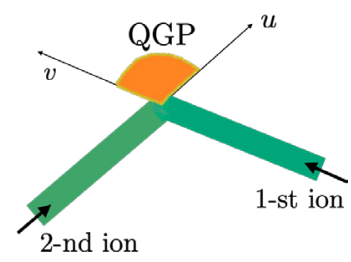

B

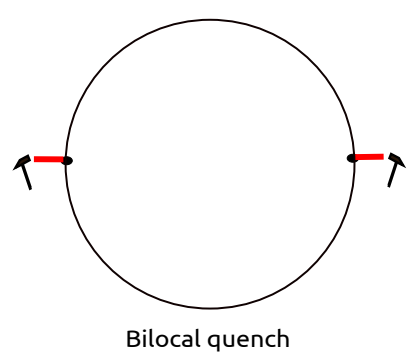

D

Figure 6. A. A schematic picture of two shock waves collision and black hole formation in $A d S_{5}$. B. A schematic picture of two ions collision and QGP formation in $(1+3)$ space-time. C. A schematic picture of two ultrarelativistic particles collision in $A d S_{5}$. D. A schematic picture of two quenches the $(1+1)$ dimensional conformal field theory.
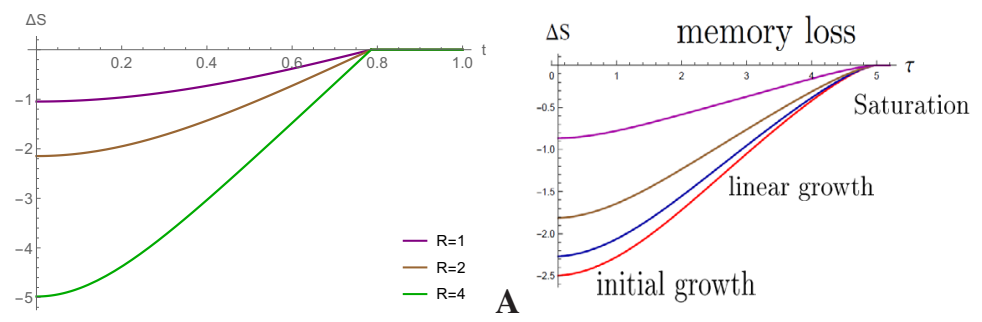

B

Figure 7. Universality of entanglement growth: A. bilocal quench; B. global quench case, plot from [23].

\section{Multiplicity in $(1+3)$ and area of the trapped surface in $(1+4)$}

The experimental total multiplicity dependence on energy [25] (Fig.9) is

$$
\mathcal{M}_{L H C} \sim s^{0.155(4)} \text {. }
$$

There were several attempts to reproduce this dependence (7) within the holographic approach in the literature (see for review $[9,10]$ and refs therein). Initially this problem was 


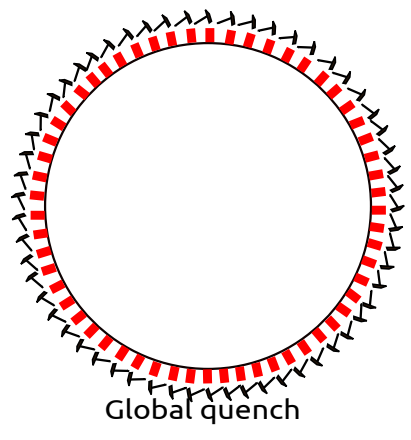

A

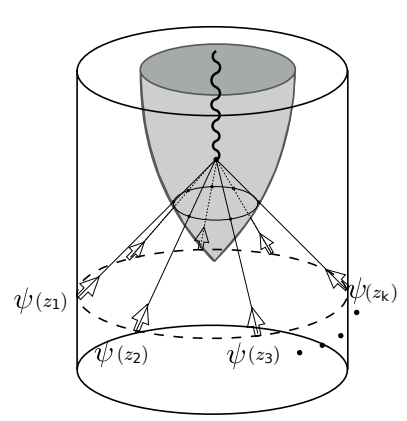

B

Figure 8. A. A schematic picture of global thermalization in the (1+1)-dimentional boundary theory. B. A schematic picture of $\mathrm{BH}$ formation after a global quench, the plot is taken from [24].

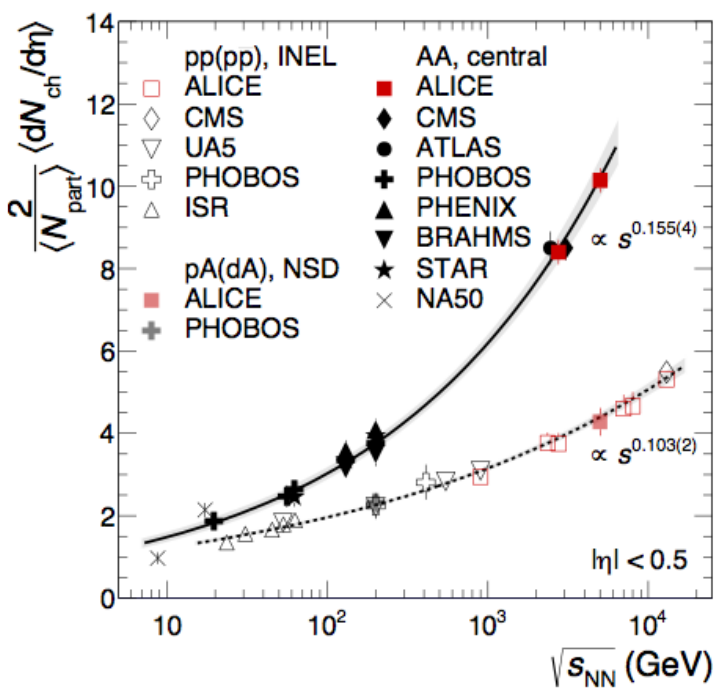

Figure 9. Values of $\frac{2}{N_{\text {part }}}\left\langle d N_{c h} / d \eta\right\rangle$ for central $\mathrm{Pb}-\mathrm{Pb}$ and $\mathrm{Au}-\mathrm{Au}$ collisions as a function of $\sqrt{s_{N N}}$. Plot from [25] (ALICE).

considered in AdS background or its isotropic deformations [26-34, 36, 37], and the total multiplicity within this approach was estimated as

$$
\mathcal{M}_{\text {AdS }} \sim s^{0.33} \text {. }
$$

For the improved holographic background the estimation was [35]

$$
\mathcal{M}_{I H Q C D} \sim s^{0.22}(1+\log \text { corrections }) .
$$


In [13] it was shown that the dependence (7) can be reproduced in the anisotropic background, namely, in the background of the form

$$
d s^{2}=\frac{L^{2}}{z^{2}}\left[-d t^{2}+d x^{2}+\left(\frac{z}{L}\right)^{2-\frac{2}{v}}+d z^{2}\right] .
$$

In the background (10) the estimation in [13] gives

$$
\mathcal{M}_{v} \sim s^{1 /(v+2)}
$$

and therefore to get (7) we take $v=4.45$.

\section{Anisotropic Holography}

One can think that QGP is anisotropic for a short time. The time of local isotropization is about $\tau_{\text {iso }} \sim 2 \mathrm{fm} / \mathrm{c}$. A schematic picture of anisotropic thermalization is displayed in Fig. 10 .

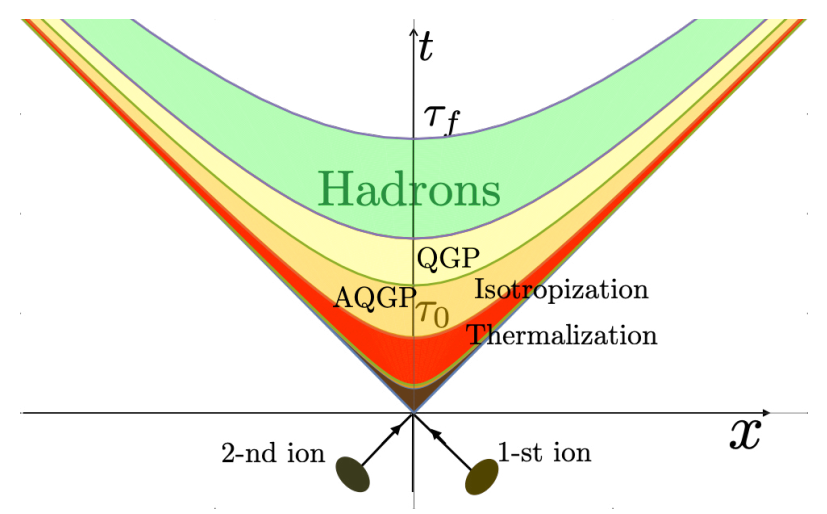

Figure 10. A schematic picture of anisotropic thermalization.

In Fig. 11 we present phase diagrams in the $(T, \mu)$-plane for the isotropic background (green line) and for anisotropic $v=4.5$ background (blue line). LIBH and SIBH (LABH and $\mathrm{SABH}$ ) indicate the regions of small and large isotropic (anisotropic) black holes. In the same plot we present also the diagram for the isotropic background (purple line) and for anisotropic $v=4.5$ background (magenta line) for model with different $b(z)$, namely for $b(z)$ proposed for isotropic case in [38].

An explanation of the plot presented in Fig.11 is the following. Horizon as function of temperature can be a multivalued function, see typical examples presented in Fig.12.

For all cases the dependence of the entropy on the horizon size has a typical form presented in Fig.13.A. This is a decreasing function. But due to the Van der Waals character of temperature dependence on $z_{h}$ we get the regions of unstable temperature behavior of entropy (they are indicated by the red color on Fig.13.B). Taking into account the relation between entropy and the free energy

$$
F=\int s d T
$$

we get the free energy dependence on the temperature presented in Fig.14 for the model [15].

The temperature dependence on $z_{h}$ presented in Fig.12. D is slightly different from the dependence shown in Fig.12. B, but it causes a first order phase transition elsewhere on the $(T, \mu)$ plane, see Fig. 11. 


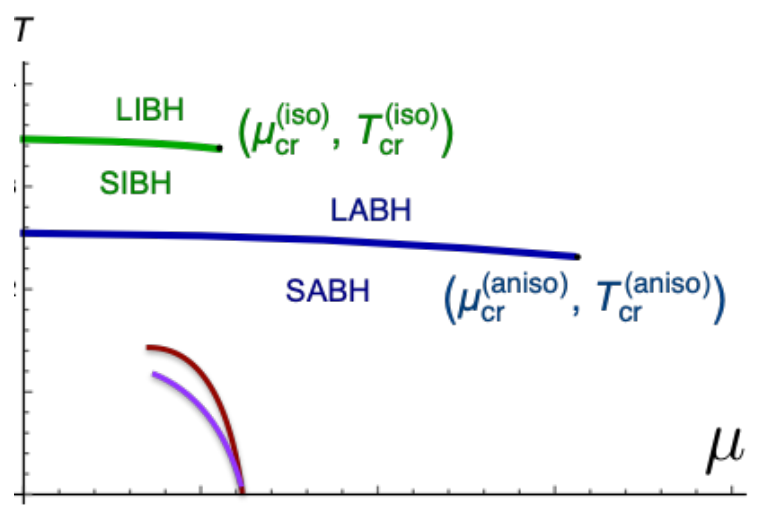

Figure 11. Phase diagrams in the $(T, \mu)$-plane for the isotropic background (green line) and for anisotropic $v=4.5$ background (blue line) for the model [15]. The purple line corresponds to isotropic model [38] and the magenta line to its anisotropic $v=4.5$ version.

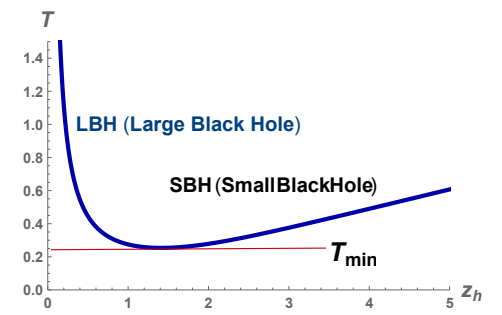

A

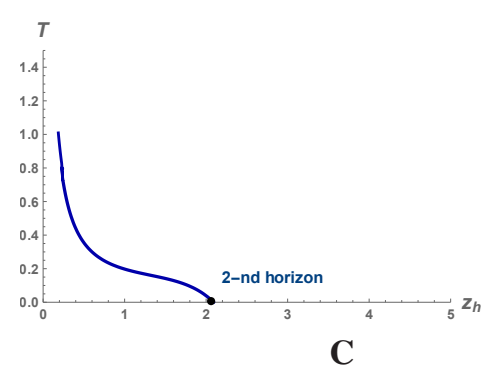

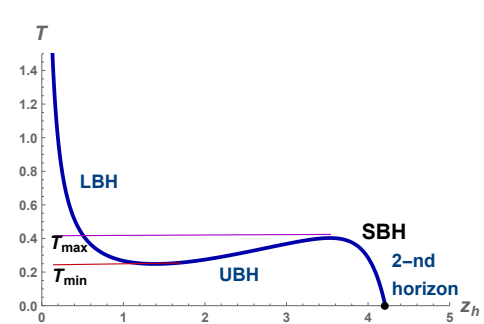

B

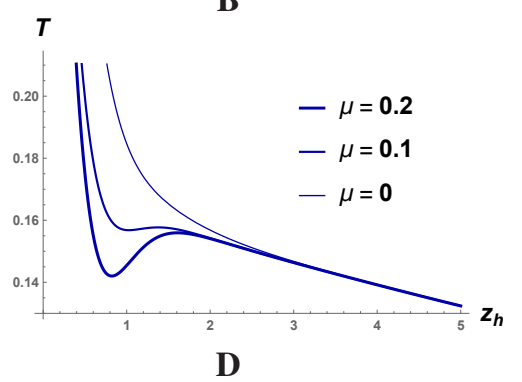

Figure 12. Dependence of temperature $T$ on the horizon size $z_{h}$ for: AdS black hole (A), the anisotropic model [15] (B for small chemical potential and $\mathbf{C}$ for large chemical potential) and for model considered in [38] (D) for different $\mu$.

\section{Conclusion}

In this talk the main ideas of holographic QCD are presented. Holographic QCD is actually a new phenomenological approach to QCD, which allows to work simultaneously in different areas of energy - from UV to IR energies. The main advantages of the holographic approach are the natural mechanisms for describing thermalization. The holographic approach allows us also to work with non-zero chemical potential. It is also gives a natural framework for dealing with anisotropy, which apparently always appears immediately after the collision of 


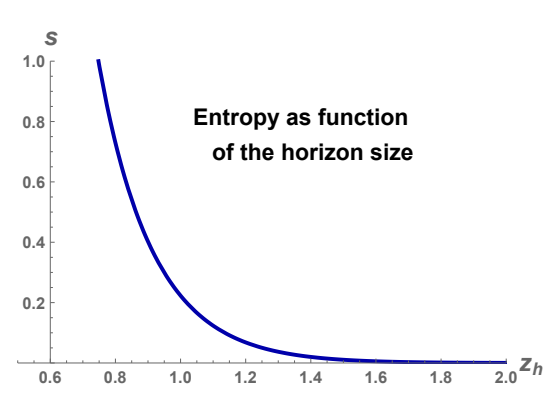

A

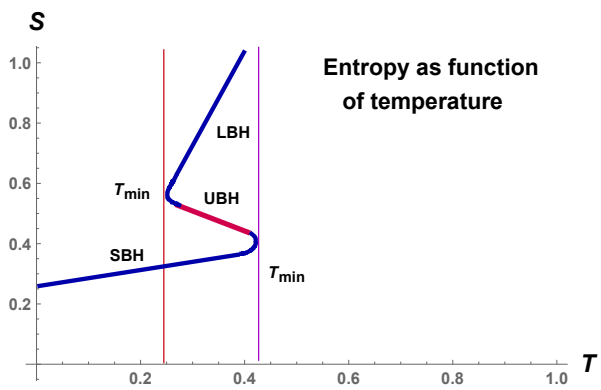

B

Figure 13. A. Dependence of entropy on $z_{h}$. B. Dependence of entropy on $T$.

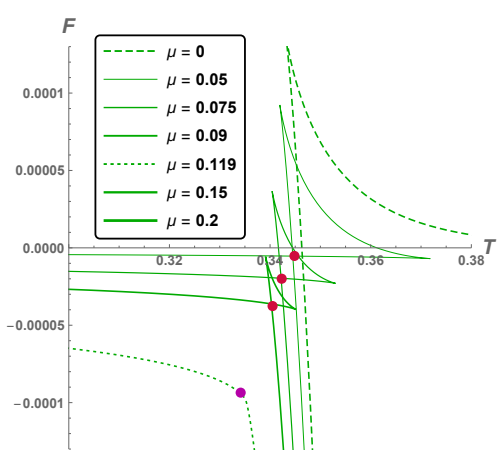

A

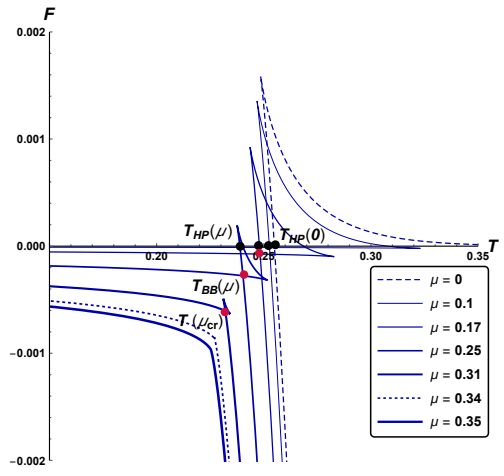

B

Figure 14. Dependence of the free energy on $T$ : A) for the isotropic case, B ) for the anisotropic case. The points of the first order phase transitions are indicated by the red points.

heavy ions. There is also anisotropy due to the appearance of a strong magnetic field in non-peripheral collisions.

\section{Acknowledgments}

This paper is based on a talk at the XXIV International Workshop High Energy Physics and Quantum Field Theory at Sochi, Russia on 22 - 29 September 2019. I would like to thank the organizers of QFTHEP'2019 for the invitation. I would like to thank D. Ageev, A. Golubtsova, M. Khramtsov, A. Patrushev, K. Rannu and P. Slepov for useful discussions. This work was supported by RFBR Grant 18-02-40069.

\section{References}

[1] A. Andronic, P. Braun-Munzinger, K. Redlich and J. Stachel, Nature 561, 321 (2018)

[2] M. Aggarwal, et al., Phys.Rev.Lett. 105022302 (2010)

[3] X. Luo, Nucl. Phys. A 904-905, 911c (2013)

[4] L. Adamczyk, et al., Phys.Rev.Lett. 112032302 (2014) 
[5] X. Luo, PoS CPOD 2014, 019 (2015)

[6] H. T. Ding, F. Karsch and S. Mukherjee, Int. J. Mod. Phys. E 24, 1530007 (2015)

[7] M. A. Stephanov, Prog. Theor. Phys. Suppl. 153, 139-156 (2004)

[8] J. Casalderrey-Solana, H. Liu, D. Mateos, K. Rajagopal and U. A. Wiedemann, Gauge/String Duality, Hot QCD and Heavy Ion Collisions, (Cambridge University Press, 2014)

[9] I. Ya. Aref'eva, Phys. Usp. 57, 527 (2014)

[10] O. DeWolfe, S. S. Gubser, C. Rosen and D. Teaney, Prog. Part. Nucl. Phys. 75, 86 (2014)

[11] M. Strickland, Pramana 84, 671 (2015).

[12] P. de Forcrand, W. Unger and H. Vairinhos, Phys. Rev. D 97, 034512 (2018)

[13] I. Ya. Aref'eva and A. A. Golubtsova, JHEP 1504, 011 (2015)

[14] I. Y. Aref'eva, A. A. Golubtsova and E. Gourgoulhon, JHEP 1609, 142 (2016)

[15] I. Aref'eva and K. Rannu, JHEP 1805, 206 (2018)

[16] I. Aref'eva, K. Rannu, P. Slepov, "Cornell potential for anisotropic QGP with non-zero chemical potential", this proceedings.

[17] I. Aref'eva, A. Patrushev and P. Slepov, Holographic Entanglement Entropy in Anisotropic Background with Confinement-Deconfinement Phase Transition, in preparation; P. Slepov, "Entanglement entropy in strongly correlated systems with confinement/deconfinement phase transition and anisotropy", this proceedings

[18] J. M. Maldacena, Phys. Rev. Lett. 80, 4859 (1998)

[19] U. Gursoy, E. Kiritsis, L. Mazzanti and F. Nitti, JHEP, 0905, 033 (2009)

[20] E. Kiritsis, W. Li and F. Nitti, Fortsch. Phys. 62, 389 (2014)

[21] I.Ya. Aref'eva, TMP, 200 , 1313(2019)

[22] I.Ya. Aref'eva, M.A. Khramtsov and M. D. Tikhanovskaya, JHEP, 9, 115 (2017)

[23] D. S. Ageev and I. Y. Aref'eva, JHEP 1803, 103 (2018)

[24] T. Anous, T. Hartman, A. Rovai and J. Sonner, JHEP 1607, 123 (2016)

[25] J. Adam et al. [ALICE Collab.], Phys. Rev. Lett. 116, 222302 (2016)

[26] S. S. Gubser, S. S. Pufu and A. Yarom, Phys. Rev. D 78, 066014 (2008)

[27] S. S. Gubser, S. S. Pufu and A. Yarom, JHEP 0911, 050 (2009)

[28] L. Alvarez-Gaume et al., JHEP 0902, 009 (2009)

[29] S. Lin and E. Shuryak, Phys. Rev. D 79, 124015 (2009)

[30] J. L. Albacete, Y. V. Kovchegov and A. Taliotis, JHEP 0905, 060 (2009)

[31] I. Ya. Aref'eva, A. A. Bagrov and E. A. Guseva, JHEP 0912, 009 (2009)

[32] I. Ya. Aref'eva, A. A. Bagrov and L. V. Joukovskaya, JHEP 1003, 002 (2010)

[33] Y. V. Kovchegov and S. Lin, JHEP 1003, 057 (2010)

[34] Y. V. Kovchegov, Prog. Theor. Phys. Suppl. 187, 96 (2011)

[35] E. Kiritsis and A. Taliotis, JHEP 1204, 065 (2012)

[36] I. Ya. Aref'eva, E. O. Pozdeeva and T. O. Pozdeeva, TMP, 176, 861 (2013)

[37] D. S. Ageev and I. Ya. Aref'eva, J. Exp. Theor. Phys. 120, 436 (2015)

[38] M. W. Li, Y. Yang and P. H. Yuan, Phys. Rev. D 96, 066013 (2017) 